\title{
Brain gray matter volume changes associated with motor symptoms in patients with Parkinson's disease
}

De-zhi Kang ${ }^{1 *}$, Fu-yong Chen ${ }^{1}$, Fang-yu Wang ${ }^{1}$, Guo-rong Wu², Ying Liư ${ }^{3}$, Gang Wu', Liang-hong Yu', Yuan-xiang Lin ${ }^{1}$ and Zhang-ya Lin ${ }^{1}$

\begin{abstract}
Background: Parkinson's disease (PD) is a common neurodegenerative disease. Most studies have found that the histopathological lesion is not only localized at the extrapyramidal area (basal ganglia) but also at the cortex in PD patients. Voxel-based morphometry (VBM) based on the voxel as a unit is described for quantitative detection of density and volume of brain tissue. In this study, VBM was used to investigate the brain gray matter changes associated with motor symptoms in PD patients.

Methods: Twelve outpatients with PD and 12 healthy controls were recruited in our hospital from September 2013 to March 2014. VBM was performed on the whole brain of all subjects. Image processing and statistical analysis were performed using SPM8. A two-sample $t$ test and multiple regression analysis were performed. Results were displayed with a threshold of $P<0.01$, corrected by false discovery rate (FDR) correction and cluster size $>30$ voxels.

Results: Comparing control healthy subjects with the patients, the data showed that PD patients had reduced gray matter volume in the postcentral gyrus, the right supramarginal center, superior temporal gyrus, precentral gyrus, Brodmann area 41, transverse temporal gyrus, Brodmann area 3, and inferior parietal lobule. The data also found that between gray matter volume and UPDRSIII in PD patients, there were negative correlations in the right middle frontal gyrus, BA06, right precentral gyrus, right superior frontal gyrus, and medial frontal gyrus, and between gray matter volume and Hoehn-Yahr (HY) in PD patients, there were negative correlations in the right middle frontal gyrus, right superior frontal gyrus, BA6, and right precentral gyrus.
\end{abstract}

Conclusions: These data supported that extensive changes associated with motor symptoms in the gray matter volume was mainly located in the related area of movement, which had obvious relevance with the progression of PD.

Keywords: Parkinson's disease, Gray matter volume, Voxel-based morphometry, Magnetic resonance imaging

\footnotetext{
* Correspondence: kdz99988@sina.com

${ }^{1}$ Department of Neurosurgery, The First Affiliated Hospital, Fujian Medical

University, Fuzhou 350005, Fujian Province, China

Full list of author information is available at the end of the article
} 


\section{Background}

Parkinson's disease which was first described by the British physician James Parkinson in 1817 is a common neurodegenerative disease, mainly in the elderly. It is estimated that the morbidity rate was $1.7 \%$ in the elderly over the age of 65 , and PD patients are 1.7 million over the age of 55 in our country [1]. PD results from the degeneration and loss of dopaminergic neurons and the forming of Lewy body. Because of the lack of clear imaging diagnosis in clinical studies, more and more scholars begin to find out the imaging changes in PD with the development of modern imaging technologies.

The ways to study the macrostructure of the brain by magnetic resonance imaging (MRI) are VBM and hand-drawn regions of interest (ROIs), which can be used to find out the change of cortex and subcortical structures in PD patients. The VBM was first formally proposed in 2000 by the British scientist Ashburner [2], which is immediately concerned by many scholars, now has been widely used in the analysis of brain structure differences. VBM is a morphometry method as voxels for the unit, which should be used after all individuals in the study group of brain MRI gradient echo T1-weighted image are spatial normalized to an identical 3D space, and then the highresolution, high-definition, high gray matter of brain structure images anatomically divide into gray matter, white matter, and cerebrospinal fluid. The individually segmented components of brain tissue are analyzed between each voxel group by a parametric statistical test so that the density and volume of brain gray matter and white matter can be detected quantitatively, and the anomaly of brain morphology was analyzed quantitatively. Spatial normalization, brain tissue segmentation, smoothing, statistical modeling, and hypothesis testing-these several steps are the processes. The advantages of VBM compared with ROI measurements is more automation, comprehensiveness, objectivity, and repeatability. Nagano-Saito et al. [3] found the gray matter atrophy in parahippocampal gyrus and inferior frontal gyrus in PD patients by VBM. Geng et al. [4] found that the putamen volume decreases in advanced PD by the use of ROI method, showing negative relation with Hoehn-Yahr staging.

In this study, VBM was used to investigate the brain gray matter changes associated with motor symptoms in PD patients.

\section{Methods}

\section{Clinical and demographic testing of samples}

Twelve right-handed PD patients including 11 males and 1 female were recruited from the Department of Neurology in The First Affiliated Hospital of Fujian Medical University, excluding other diseases. The PD patients
Table 1 The clinical information of PD patients and NCS

\begin{tabular}{lll}
\hline Index & PD patients & NCs \\
\hline Age (year) & $66.00 \pm 4.22$ & $63.17 \pm 4.11$ \\
Gender (male/female) & $11 / 1$ & $11 / 1$ \\
Disease course (year) & $7.75 \pm 4.78$ & - \\
HY & $2.58 \pm 0.90$ & - \\
UPDRSIII & $20.58 \pm 8.85$ & - \\
\hline
\end{tabular}

were diagnosed by the UK Parkinson's Disease Society Brain Bank clinical diagnosis of idiopathic Parkinson's disease standard, and excluding other diseases. There was no statistically significant difference in age and gender between the right-handed normal controls and PD patients, and all the participants were willing to participate in this research after signing informed consent. All patients came in off all medications for at least $6 \mathrm{~h}$ for imaging and testing. The general situation such as age, gender, education level, medications, and disease course was registered. Unified Parkinson's Disease Rating Scale (UPDRS) and Hoehn and Yahr (HY) were also recorded for describing the severity of the PD. The study was approved by the local Ethics Committee of The First Affiliated Hospital of Fujian Medical University, and written informed consent was obtained from all patients and healthy volunteers prior to the examination.

\section{MRI data acquisition}

VBM is based on high-resolution structural 3D MR images and is designed to investigate significant regional differences with voxel-wise statistics in the context of Gaussian random fields. MRI was performed on a Siemens scanner operating at 3.0 T. A 3D structural MRI was acquired for each subject using a T1-weighted gradient echo MP-RANGE sequence (TR $2530 \mathrm{~ms}$; TE $3.39 \mathrm{~ms}$, flip angle $=7^{\circ}$, thickness $=1.33 \mathrm{~mm}$, space $=$ $0.5 \mathrm{~mm}$, FOV $256 \times 256 \mathrm{~mm}$, matrix $=256 \times 192)$. Conventional T1 MR imaging showed no morphological

Table 2 The decreased gray matter volume in PD patients

\begin{tabular}{lll}
\hline Brain regions & BA & Cluster size \\
\hline Right postcentral gyrus & $3 / 1 / 2$ & 382 \\
Right supramarginal center & 40 & 275 \\
Right superior temporal gyrus & 22 & 205 \\
Precentral gyrus & 4 & 120 \\
Right transverse temporal gyrus & 41 & 95 \\
& 3 & 42 \\
Inferior parietal lobule & $39 / 40$ & 50 \\
\hline
\end{tabular}

BA Brodmann area 

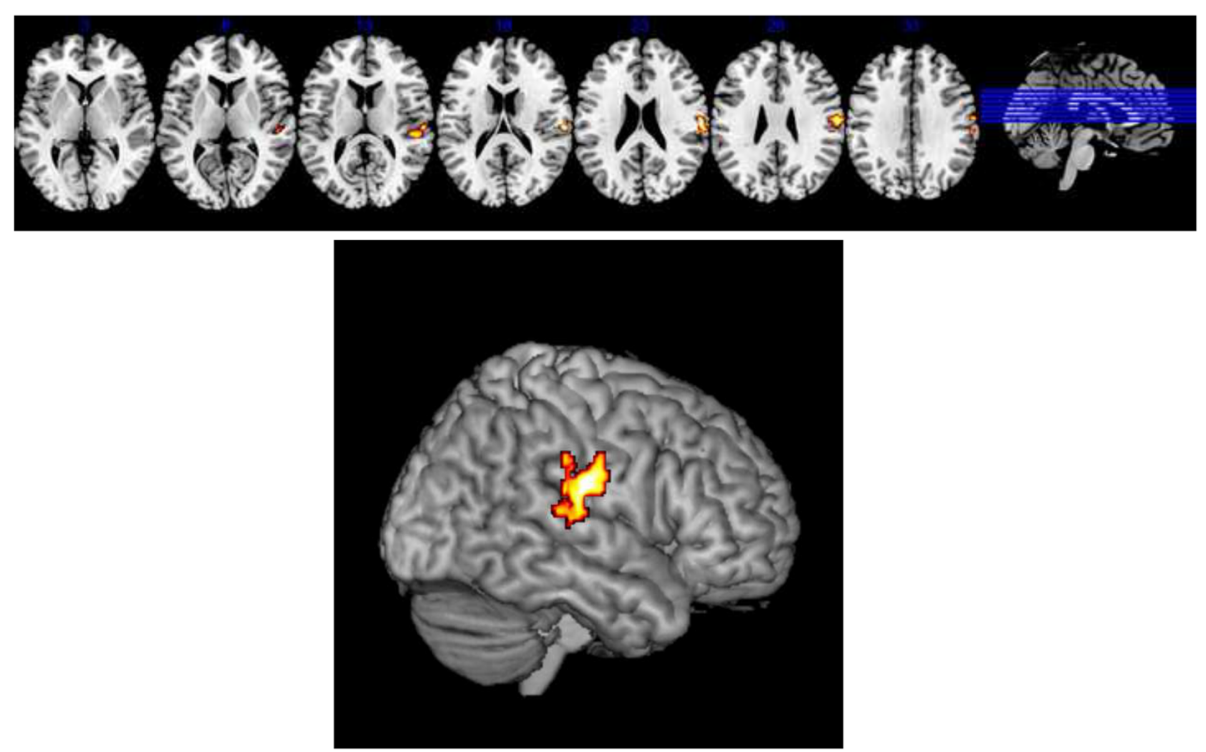

Fig. 1 The decreased gray matter volume in PD patients. The threshold for display was set to $P<0.01$, cluster $>30$, corrected by FDR

abnormalities or artifacts in the patients and health controls.

\section{Data processing and statistical analysis}

Data preprocessing and analysis were performed with SPM8 (Welcome Department of Imaging Neuroscience, London, UK) running under Matlab (Mathworks, Sherborn, MA, USA). Preprocessing of the data included spatial normalization, segmentation, modulation, and spatial smoothing with Gaussian kernel.

The images aligned with the stereotactic space defined by the Montreal Neurological Institute (MNI) were corrected for non-uniformities in signal intensity and partitioned into gray matter, white matter, and cerebrospinal fluid. Subsequently, all images were smoothed by an isotropic Gaussian kernel of $8 \mathrm{~mm}$ full-width at half maximum (FWHM).

Voxel-by-voxel two-sample $t$ test using the general linear model (GLM) was used to test for regionally specific gray matter difference between groups. Findings were considered significant at a voxel-level of $P<0.01$, corrected for FDR. Voxel-based multiple regression analysis was performed by SPM8 with voxel-wise gray matter volume as a dependent variable and UPDRSIII and HY scores as a covariate of interest.

\section{Results}

\section{Clinical and demographic characteristics}

There were no statistically significant differences in gender, age, and education level between PD patients and normal controls (NCs) (Table 1). The UPDRSIII score range was between 9 and 33 points, and the disease course ranged from 3- to 20-year duration. The clinical manifestations of PD patients were tremor, stiffness, rigidity, and both.

The difference of the two groups in gray matter volume Compared to the NCs, PD patients exhibited a decreased gray matter volume in the right postcentral gyrus, right supramarginal center, right superior temporal gyrus, precentral gyrus, BA41, right transverse temporal gyrus, BA3, and inferior parietal lobule and no increased volume of gray matter (two-sample $t$ test, $P<0.01$, false discovery rate (FDR) corrected) (Table 2, Fig. 1).

\section{Correlations between UPDRSIII score, Hoehn-Yahr, and gray matter volume}

Between gray matter volume and UPDRSIII in PD patients, there were negative correlations in the right middle frontal gyrus, BA06, right precentral gyrus, right

Table 3 The negative correlation between gray matter volume and UPDRSIII score

\begin{tabular}{lll}
\hline Negatively related brain regions & BA & Cluster size \\
\hline Right middle frontal gyrus & 6 & 260 \\
& 6 & 134 \\
Right precentral gyrus & 4 & 108 \\
Right superior frontal gyrus & & 80 \\
Medial frontal gyrus & & 34
\end{tabular}

BA Brodmann area 


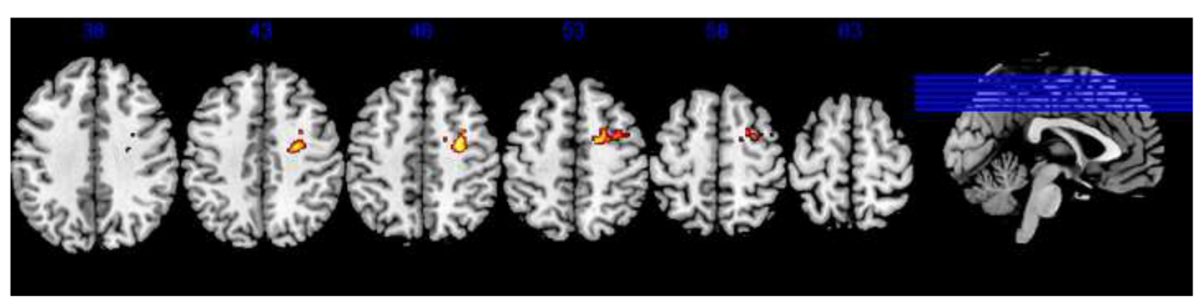

Fig. 2 Negatively correlated brain region between gray matter volume and UPDRSIII in PD patients. The threshold for display was set to $P<0.01$, cluster $>30$, corrected by FDR

superior frontal gyrus, and medial frontal gyrus $(P<0.01$, FDR correction) (Table 3, Fig. 2).

Between gray matter volume and Hoehn-Yahr (HY) in PD patients, there were negative correlations in the right middle frontal gyrus, right superior frontal gyrus, BA6, and right precentral gyrus $(P<0.01$, FDR correction) (Table 4, Fig. 3).

\section{Discussion}

MRI neuroimaging can study the changes of brain structure on the human body, and the main research methods are ROI method and VBM method. ROI method is the process that delineates the interested area firstly, then extracts the volume or density information of the region from each subject, and finally picks the differences of ROI up through statistical methods. It has the advantage of dividing accurate brain structure; however, too much human intervention and subjectivity is the disadvantage, and different classified criteria of ROI will seriously affect the final result, which is difficult to compare between different studies. The study of brain structure is moving forward with the use of VBM method. The image of each subject is registered to a standard template, and then a researcher can look for differences in the brain regions without any prior assumptions [5, 6]. This method has been used after the beginning of this century firstly, although the accuracy has been questioned and controverted, is recognized and increasingly widely used, and has become commonly used in the field of neural cognitive

Table 4 The negative correlation between gray matter volume and UPDRSIII score

\begin{tabular}{lll}
\hline Negatively related brain regions & BA & Cluster size \\
\hline Right middle frontal gyrus & & 171 \\
Right superior frontal gyrus & & 124 \\
& BA6 & 114 \\
Right precentral gyrus & BA4 & 40 \\
\hline
\end{tabular}

BA Brodmann area science as its simple operation and objective [7, 8]. But the shortcomings of this method are that only structural changes in the brain regions have been found and whether these specific brain areas can still distinguish between patients and normal subjects is unknown. DARTEL algorithm used in this study is a new method in 2007 by SPM Crown, which employs tens of thousands of parameters to standardize the shape of each brain and improve the image accuracy in different individuals and operational simplicity [9].

Parkinson's disease results from the degeneration and loss of dopaminergic neurons and the forming of Lewy bodies (Lewy body) [10], which have been confirmed by pathological studies; however, PD lesions are not confined in the nigra, and with the progress of the disease, the pathological changes gradually to the limbic system and extensive neocortex, because of the formation of Lewy bodies in local neurons, the loss and necrosis of neuron, so that the brain morphology changes.

In this study, we found that gray matter volume was extensively decreased in PD patients, which conform to the pathological mechanism in PD patients. Dyskinesia is a typical clinical manifestation of PD, and studies show that the output of extrapyramidal disorders caused by Parkinson's disease is often accompanied with changes in cortical motor function [11]. The vast majority of motor function in the cerebral cortex areas is located in the frontal, parietal cortex, mainly located in the medial frontal gyrus, postcentral gyrus, and most of the contact between the fibers in the basal ganglia and cortex are projected onto the area [11]. In this study, we found that the motor function-related brain areas appear gray matter volume reduction, and PD dyskinesia severity was negatively correlated with its decrease. Pan et al. [12] use the meta-analysis study in 17 VBM studies of Parkinson's disease brain cortical atrophy, and a comprehensive analysis found that significant regional gray matter volume reduction is mainly located on the left inferior frontal gyrus (BA47) extended to the left temporal (BA38) and left insula (BA13) in PD patients. 

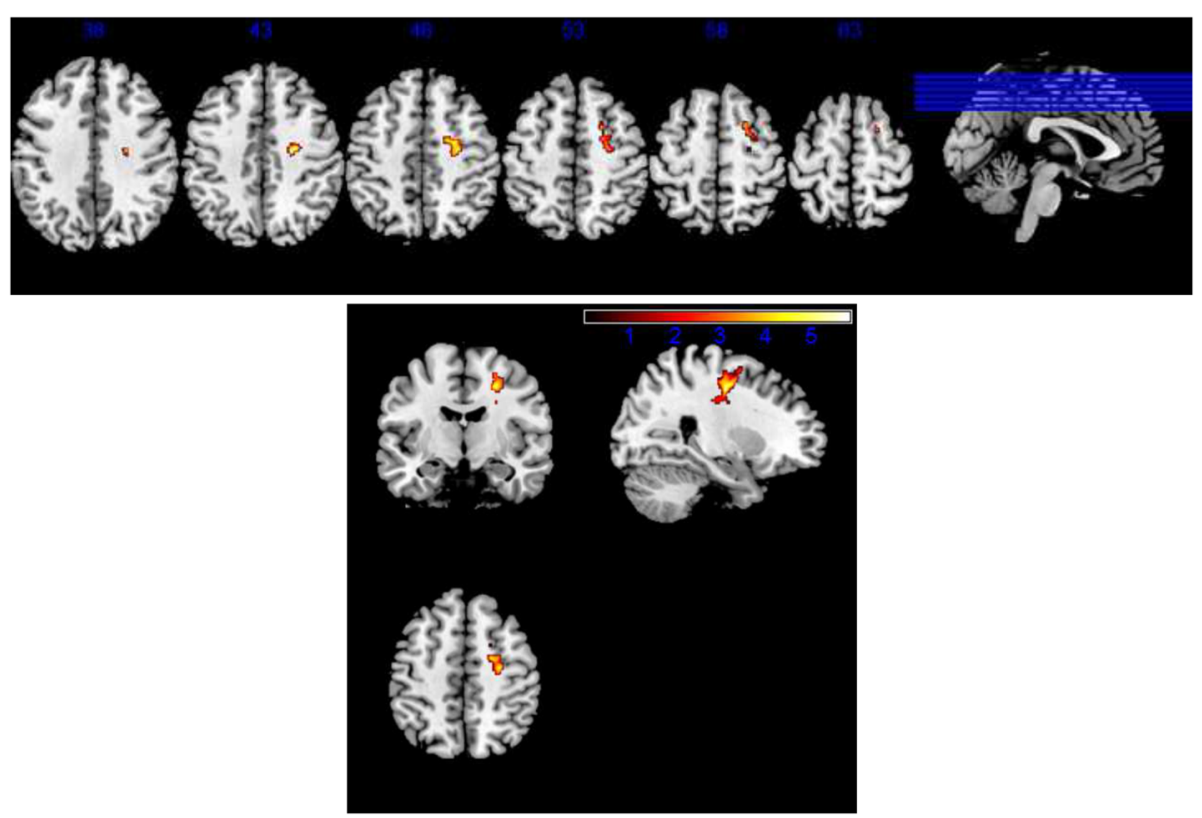

Fig. 3 Negatively correlated brain region between gray matter volume and HY in PD patients. The threshold for display was set to $P<0.01$, cluster $>30$, corrected by FDR

There is an abnormality on brain structure imaging in PD, which may be helpful to early diagnosis and prognosis in $\mathrm{PD}$.

\section{Conclusions}

We found that the change of brain gray matter structures associated with motor symptoms in PD patients was mainly located in sports-related area and had a relationship with the progression of the disease by VBM.

\section{Abbreviations}

BA: Brodmann area; FDR: false discovery rate; FOV: field of view; FWHM: full-width at half maximum; HY: Hoehn-Yahr; MNI: Montreal Neurological Institute; MP-RANGE: magnetization-prepared rapid-acquisition gradient echo; MRI: magnetic resonance imaging; NC: normal control; PD: Parkinson's disease; ROI: region of interest; SPM: statistical parameter mapping; TE: echo time; TR: reaction time; UPDRS: Unified Parkinson's Disease Rating Scale; VBM: voxel-based morphometry.

\section{Competing interests}

The authors declare that they have no competing interests.

\section{Authors' contributions}

DK conceived and designed the experiments. FC and GuW analyzed the data. FW and FC wrote the paper. GaW, LY, YL, and ZL collected the PD patients and normal control and scaled the clinical rating scale. YL carried out the MRI scan. All authors read and approved the final manuscript.

\section{Acknowledgements}

This research was supported by a grant from Key Project of Science and Technology of Fujian Province (No. 2012Y0027) and Fujian Province Natural Science Foundation (No. 2013J01298). This research was also sponsored by key clinical specialty discipline construction program of Fujian, PRC.

\section{Author details}

'Department of Neurosurgery, The First Affiliated Hospital, Fujian Medical University, Fuzhou 350005, Fujian Province, China. ${ }^{2}$ Key Laboratory of
Personality and Cognition, Faculty of Psychology, Southwest University, Beibei, Chongqing 400715, China. ${ }^{3}$ Department of Radiology, The First Affiliated Hospital, Fujian Medical University, Fuzhou 350005Fujian Province, China.

Received: 31 May 2015 Accepted: 11 June 2015

Published online: 18 August 2015

\section{References}

1. Zhang ZX, Roman GC, Hong Z, Wu CB, Qu QM, Huang JB, et al. Parkinson's disease in China: prevalence in Beijing, Xian, and Shanghai. Lancet. 2005;365(9459):595-7.

2. Ashburner J, Friston KJ. Voxel-based morphometry-the methods. Neuroimage. 2000;11(6):805-21

3. Nagano-Saito A, Washimi Y, Arahata Y, Kachi T, Lerch JP, Evans AC, et al. Cerebral atrophy and its relation to cognitive impairment in Parkinson disease. Neurology. 2005;64(2):224-9.

4. Geng D, Li YX, Zee CS. Magnetic resonance imaging-based volumetric analysis of basal ganglia nuclei and substantia nigra in patients with Parkinson's disease. Neurosurgery. 2006;58(2):256-62.

5. Good CD, Johnsrude IS, Ashburner J, Henson RN, Friston KJ, Frackowiak RS, et al. A voxel-based morphometric study of ageing in 465 normal adult human brains, Biomedical Imaging, 2002, 5th IEEE EMBS International Summer School on. IEEE. 2002. p. 16.

6. Ashburner J. Computational anatomy with the SPM software. Magn Reson Imaging. 2009:27(8):1163-74.

7. Ashburner J, Friston KJ. Why voxel-based morphometry should be used. Neuroimage. 2001;14(6):1238-43.

8. Bookstein FL. "Voxel-based morphometry" should not be used with imperfectly registered images. Neuroimage. 2001;14(6):1454-62.

9. Ashburner J. A fast diffeomorphic image registration algorithm. Neuroimage. 2007;38(1):95-113.

10. Braak H, Tredici KD, Rüb U, de Vos RA, Jansen Steur EN, Braak E. Staging of brain pathology related to sporadic Parkinson's disease. Neurobiol Aging. 2003;24(2):197-211

11. Fukuda M, Edwards C, Eidelberg D. Functional brain networks in Parkinson's disease. Parkinsonism Relat Disord. 2001;8(2):91-4.

12. Pan $\mathrm{PL}$, Song $\mathrm{W}$, Shang HF. Voxel-wise meta-analysis of gray matter abnormalities in idiopathic Parkinson's disease. Eur J Neurol. 2012;19(2): 199-206. 